\title{
Vehicle Energy Management System
}

\author{
Z. Burě̌ \\ Department of Communication and Information Systems, University of Defence, Brno, Czech Republic \\ *Corresponding author: zbynek.bures@unob.cz
}

M. Šula

MSR Engines Ltd., Brno, Czech Republic

V. Přenosil

Department of Informatics Technology, Faculty of Informatics, Masaryk University, Brno, Czech Republic,

DOI: $10.2478 / \mathrm{v} 10158-012-0006-3$

\begin{abstract}
The paper deals with power management systems in a vehicle comprising of an internal combustion engine which is connected via a gearbox with a clutch and transmission to a main generator of electricity. The battery and supercapacitors, as the central independent power source, are connected via the control module and via a bidirectional communication bus to the vehicle state evaluation unit. Both modes charging and discharging of the battery and supercapacitors together with alternator control is the most affected by the evaluation unit.
\end{abstract}

KEY WORDS: intelligent power system, operational energy storage - supercapacitor, automotive onboard network, control unit, vehicle energy management system.

\section{INTRODUCTION}

The arrangement of the Vehicle Energy Management System (V.E.M.S.) consists of a traditional combustion engine connected through a transmission mechanism plus clutch and a transmission mechanism to the main generator connected (through the first power lead) to the first converter, which is further connected (through the fifth power lead) to the sixth voltage converter (which is further connected to the energy storage through the sixth power lead) and to a bi-directional voltage converter. It is then connected through the seventh power lead - onboard network - to an accumulator, while its control module is connected (through a bi-directional communication bus) to a signal-converting unit, which is connected (through a communication bus) to a vehicle state evaluation unit included in the control module. Vehicle inputs are connected to both the evaluation unit and the signal-converting unit, to which a bi-directional bus and outputs are connected. The essence of the system 
is in its connection (through the third converter and the fifth power lead) to an auxiliary generator interconnected with this third converter through the third power lead, while the auxiliary generator is mechanically interconnected with a turbine inserted in the turbocharger's bypass, or with a turbine inserted into the exhaust pipe of the combustion engine.

\section{TECHNOLOGY AREA}

V.E.M.S. separates the combustion engine from providing the power for the supply of electric appliances and minimalizes the required power for the air-conditioning. This is achieved through the Electronic Intelligent Alternator, together with the Electronic Air-Conditioning, Supercapacitors, and sets of uni/bi-directional rectifiers. It also deals with the method of V.E.M.S. management, which can be described as being a hierarchical state machine with which the vehicle enters different states, illustrated in the form of different state diagrams.

\section{CURRENT TECHNOLOGY STATE}

Nowadays a maximum amount of attention is paid to complying with emission limits (the amount of $\mathrm{CO}_{2}$ produced per kilometer driven) in developing new cars. Emission reduction can be achieved through design modifications in the combustion engine itself, by reducing the vehicle weight, and by reducing the electricity consumption for times when the engine runs outside its optimum revolution range. Contrary to this trend, however, are the ever-growing requirements of the onboard electricity network. Ever-richer car features (automatic air-conditioning, heated seats, heated windscreen and side mirrors, power-steering boosts, safety systems, etc.) significantly increase the electricity consumption. Modern vehicles are equipped with high-efficiency (up to 80\%) alternators and use two electricity distribution networks? with different voltages $(12 \mathrm{~V} / 24 \mathrm{~V} / 48 \mathrm{~V})$. The transition to a higher voltage brings with it a number of benefits arising from the reduced weights of power harness and components (starter, alternator, pumps). Unfortunately, these modifications do not eliminate the high electricity drain from the alternator during moving off after engine starting, during vehicle acceleration, or during steady driving. Electricity is currently produced only by transforming the fuel's chemical energy into mechanical energy by means of combustion within an engine, and this is further transformed into electrical energy by means of an alternator. The power taken from the engine by the alternator manifests itself in the engine's increased nominal consumption and in the amount of $\mathrm{CO}_{2}$ produced, which is directly related to it. This can be partly solved by combining a combustion engine and an electric motor, i.e., by "hybrid propulsion". Even vehicles with hybrid propulsion, however, struggle with two limiting factors: 1 . the battery - a structural element with a slow charging cycle, great weight and great volume, and 2. the weight of the whole system (around $200 \mathrm{~kg}$ ). Thanks to these factors, such vehicles are economical when operated with frequent and long decelerations (i.e., driven in cities), but have a great disadvantage when operated on motorways, where they feature increased consumption due to the higher vehicle weight and due to the battery being charged mainly from the combustion engine (from petrol). A complete solution of the exhalation and consumption problems is provided by electric cars. Their utilization, however, is limited by battery technologies. Due to their weight, means of charging, price, and environmental impact, today's batteries cannot provide a technical solution with parameters comparable to conventional propulsion. 
Apart from deceleration, exhaust gas is another energy source in combustion engine vehicles. Current vehicles do not fully utilize the energy contained in the exhaust gas. The only currently utilized energy is a small portion used for propelling the turbocharger compressing the air in the suction pipeline in turbo vehicles. The remaining large amount of energy is released through the exhaust pipe into the atmosphere.

\section{ESSENCE OF THE INVENTION}

The objective of the invention is to propose a new arrangement of the vehicle energy management system (V.E.M.S.), as well as a method for its control to reduce the fuel consumption and thus the $\mathrm{CO}_{2}$ emissions produced. The vehicle's electrical management combines and controls the following mechanical/electrical vehicle components: the turbocharger or turbo compressor connected to the combustion engine, to which an actuator (motor generator) is connected, as well as an intelligent alternator (described in the patent application No. PV (286-2009), the main electric motor (booster), electronic air-conditioning and an electricity storage (super-capacitors or batteries).

The above weaknesses are eliminated by arranging the V.E.M.S. consisting of a combustion engine connected through a transmission mechanism plus clutch and a transmission mechanism to the main generator connected (through the first power lead) to the first converter, which is further connected (through the fifth power lead) to the sixth voltage converter (which is further connected to the energy storage through the sixth power lead) and to a bi-directional voltage converter. Then it is connected through the seventh power lead - onboard network - to an accumulator, while its control module is connected (through a bi-directional communication bus) to a signal-converting unit, which is connected (through a communication bus) to a vehicle state evaluation unit included in the control module. Vehicle inputs are connected to both the evaluation unit and the signal-converting unit, to which a bi-directional bus and outputs are connected. The essence of the system is its connection (through the third converter and the fifth power lead) to an auxiliary generator interconnected with this third converter through the third power lead, while the auxiliary generator is mechanically interconnected with a turbine inserted into the turbocharger's bypass or with a turbine inserted into the exhaust pipe of the combustion engine.

To reduce the combustion engine consumption, it is beneficial if the fifth converter is connected to the fifth power lead and further interconnected with an electric motor, which is mechanically connected to the compressor of the air-conditioning unit.

To improve the combustion engine's acceleration and to reduce its emissions while in transition modes, it is beneficial to have the main electric motor connected to the fifth power lead through the second converter, and further connected to the combustion engine through a transmission mechanism and a transmission mechanism plus clutch.

To reduce the combustion engine fuel consumption and its emissions, it is beneficial to have the main electric motor connected to the fifth power lead through the second converter, and further connected to the combustion engine through a transmission mechanism and a transmission mechanism plus clutch.

To reduce weight and to simplify insertion of a motor and a generator into the engine compartment, it is beneficial if the main electric motor forms an integral part of the main generator and the second converter forms an integral part of the first converter.

To improve the turbocharger's dynamics, it is beneficial if the auxiliary electric motor is connected through the fifth power lead and the fourth converter, and further connected to this fourth converter through the forth power lead and mechanically connected to the turbocharger shaft. 


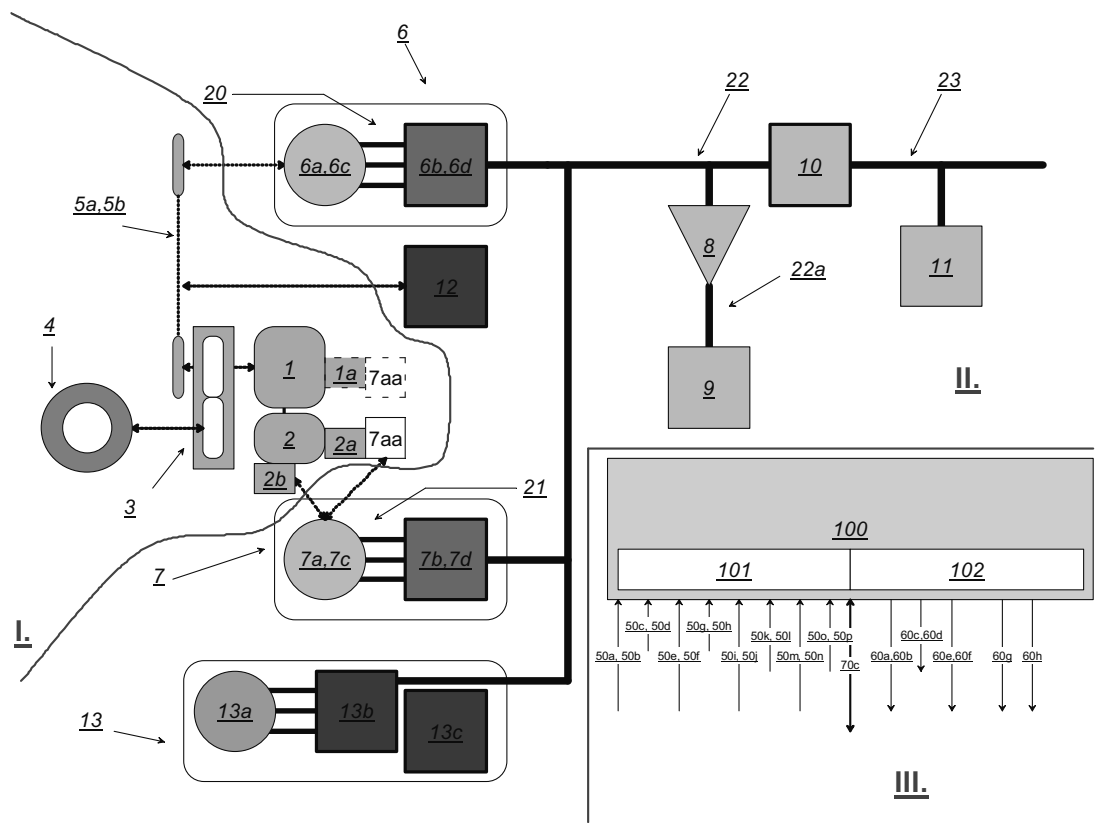

Legend of Reference Points

\section{- Mechanical part \\ III - Control system \\ 1 - Combustion engine \\ $1 \mathrm{a}$ - exhaust system}

2 - combustion engine's turbocharger

$2 a-$ bypass for turbocharger's exhaust gas (waste gate),

$$
2 b \text { - turbocharger's shaft, }
$$

3 - Transmission mechanism+clutch,

\section{4 - Driven axle}

$5 a-R p m-c h a n g i n g$ transmission (for generator)

$5 \mathrm{~b}-\mathrm{Rpm}$-changing transmission (for electric motor),

6 - Set - motor-generator (actuator)

$6 a$ - Main generator

$6 \mathrm{~b}-$ First converter (isolating switch),

$6 \mathrm{c}$ - Main electric motor,

$6 \mathrm{~d}-$ Second converter (controller),

7 - Set - turbo-engine-generator

$7 a-$ Auxiliary generator

$$
\text { 7aa - Turbine }
$$

$7 \mathrm{~b}-$ Third converter (isolating switch)

7c - Auxiliary electric motor,

$7 \mathrm{~d}$ - Fourth converter (controller)

8 - Sixth converter (isolating switch for energy storage)

9 - Energy storage (super-capacitors or batteries),

10 - Bi-directional voltage converter or two single-directional voltage converters

11 - Onboard accumulator

12 - Adjusting mechanism of rpm-changing transmission,

13 - Set - electronic air-conditioning,

$13 a-$ Third electric motor for air-conditioning

compressor

$13 b-$ Fifth converter (controller),

$13 \mathrm{c}$ - Controlling electronics for electronic air-

conditioning

20 - Power lead to main motor-generator (main generator, booster),

$20 \mathrm{a}$ - First three-phase power lead (generator / converter),

$20 \mathrm{~b}$ - Second three-phase power lead (converter / electric motor),

21 - Power lead to auxiliary motor-generator (turbocharger) $21 \mathrm{a}$ - Third three-phase power lead (generator / converter),

$21 \mathrm{~b}$ - Fourth three-phase power lead (converter / electric motor)

22 - Fifth power lead

$22 a$ - Sixth power lead,

23 - Seventh power lead - onboard network
50 - Inputs

50 a - battery voltage 11

$50 \mathrm{~b}$ - current to/from battery 11 .

$50 \mathrm{c}$ - voltage of super-capacitors $\underline{9}$,

$50 \mathrm{~b}$ - current to/from super-capacitors $\underline{9}$,

50 e-generator rpm $6 a$,

$50 f-$ revolutions of rpm-changing transmission at connection with transmission mechanism+clutch (nodes $\underline{5}$ and 3 ),

$50 \mathrm{~g}$ - rpm of combustion engine 1 ,

$50 \mathrm{~h}-\mathrm{rpm}$ of turbocharger 2

$50 \mathrm{i}$ - combustion engine 1 temperature

$50 \mathrm{j}$ - gas throttle valve/pedal position,

$50 \mathrm{k}$ - braking system pressure or braking pedal

position,

501 - clutch pedal state

$50 \mathrm{~m}$ - input for forced energy accumulation from motor-generator $\underline{6}$ (electronic brake),

$50 \mathrm{n}$ - currently engaged gear,

$50 \mathrm{o}$ - switching on the vehicle starter,

$50 \mathrm{p}$ - ambient temperature,

$50 q$ - onboard network $\underline{23}$ off-take

60 - Outputs switch) $\underline{6 b}$

$60 \mathrm{a}$ - control signal for first converter (isolating

$\frac{6 \mathrm{~b}}{60 \mathrm{~b}}$ - control signal for second converter (controller)

6d, $60 \mathrm{c}-$ control signal for third converter (isolating switch) $\underline{7 b}$,

7d,

$60 \mathrm{~d}$ - control signal for fourth converter (controller)

$60 \mathrm{e}-$ control signal for fifth converter (controller)

$13 \mathrm{~b}$ switch) $\underline{8}$,

$60 f-$ control signal for sixth converter (isolating

$60 \mathrm{~g}$ - control signal for bi-directional converter 10 $60 \mathrm{~h}$-position control 12

70 - communication and diagnostic buses (CAN, RS485, RS232 or others),

$70 \mathrm{a}$ - bi-directional communication bus

(intermodular 100 - 101)

$70 \mathrm{~b}$ - communication bus (intermodular $101-102$ ),

$70 \mathrm{c}$ - bi-directional communication bus (CAN,

RS485, RS232 or others),

100 - Control module (unit)

101 - Signal-converting unit 100

102 - Vehicle state evaluation unit 100

Figure 1: Schematic diagram of the V.E.M.S. 
To reduce the combustion engine fuel consumption, it is beneficial if the auxiliary electric motor is connected through the fifth power lead and the fourth controller, and further connected to this fourth converter through the forth power lead and mechanically connected to the turbocharger shaft.

To reduce weight and to simplify the insertion of a motor and a generator into the engine compartment, it is beneficial if the auxiliary electric motor forms an integral part of the auxiliary generator and the fourth converter forms an integral part of the third converter.To increase the efficiency of the control module structure, it is recommended that the vehicle state evaluating unit and the signal-converting unit are integrated into it.

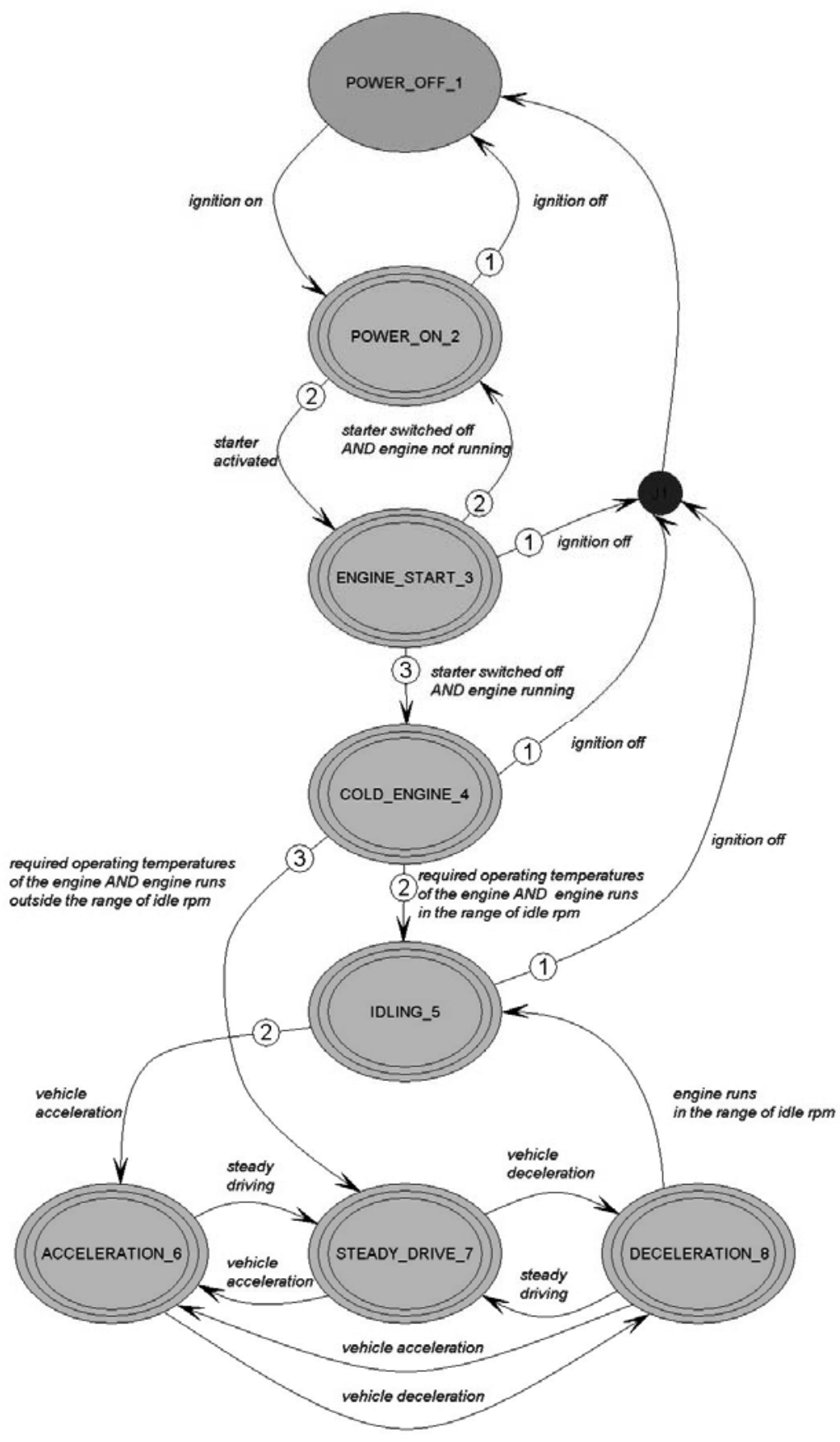

Figure 2: State diagram of the V.E.M.S. 
It is beneficial for the V.E.M.S: control if the control module is connected through a communication bus to the signal-converting unit, the outputs of which are connected to different blocks within the arrangement. The signal-converting unit is further wired to the vehicle state evaluation unit through a communication interface, while vehicle inputs are connected to the vehicle state evaluation unit and to the signalconverting unit, to which a bi-directional bus is conveniently connected.

To simplify the implementation of the control algorithms in the V.E.M.S. control module, it is beneficial if the vehicle state evaluation unit has output 1 connected to the input of the first voltage converter, output 2 to the input of the third voltage converter, output 3 to the input of the sixth converter (at the electricity storage), output 4 to the input of the bi-directional voltage converter, output 5 to the input of the module setting the correct rpm ratio of the transmission mechanism, and output 6 to the input of the fifth converter (at the electronic air-conditioning). The signal-converting unit should have its inputs 1 and 2 connected to the accumulator outputs, inputs 3 and 4 to outputs of the electricity storage (super-capacitors or batteries, for example), input 5 to the main generator's rpm sensor, input 6 to the rpm sensor of the rpm-changing transmission mechanism installed at the link to the transmission mechanism plus clutch. Input 7 should be connected to the combustion engine's rpm sensor, input 8 to the turbocharger's rpm sensor, input 9 to the engine's temperature sensor, input 10 to the accelerator pedal position sensor, input 11 to the pressure sensor of the vehicle's braking system, input 12 to the clutch pedal position sensor, input 13 to the input of the forced accumulation of the energy from the motor-generator, input 14 to the gear sensor, input 15 to the starter switch sensor, input 16 to the ambient temperature sensor, and input 17 to the onboard network off-take sensor. To make system diagnostics possible, the control module is also connected to the vehicle control unit through a communication/diagnostic bus (this connection is not shown in diagrams).

The key is the arrangement of the V.E.M.S., which better utilizes the energy in the vehicle as a whole. Thanks to gaining energy from the exhaust gas and from deceleration, and to its logical distribution between the electronic air-conditioning, main electric motor (booster), auxiliary electric motor (for faster turbocharger start-up) and the onboard network power supply, significant reductions in fuel consumption, as well as $\mathrm{CO}_{2}$ emissions, are achieved at the current weight of the system below $25 \mathrm{~kg}$. The main philosophy of the V.E.M.S. is to use the combustion engine only for driving the vehicle, while all the vehicle's peripherals would be powered by electricity generated from other sources. This is associated with: "electronic air-conditioning", i.e., an airconditioning unit driven by an electric motor powered by electricity generated from alternative sources (deceleration and exhaust gas), not by the combustion engine, as is currently common (with an increase in fuel consumption when the air-conditioning is in operation). This is an important component of the whole system significantly reducing both the fuel consumption and $\mathrm{CO}_{2}$ emissions.

Apart from the re-use of the energy from braking, the use of energy contained in the exhaust gas is another path towards reductions in consumption and $\mathrm{CO}_{2}$ emissions. Exhaust gas contains between $50 \%$ and $80 \%$ of the energy produced by the combustion engine, which is currently not fully utilized, but rather released into the atmosphere. In combustion engines equipped with a turbocharger, the solution proposes using the residual energy contained in the exhaust gas for electricity generation, or applying a turbocharger in the combustion engine's exhaust pipe - used solely for electricity generation.

The essence of the controlling method of the V.E.M.S. is in the fact that the control module 100 is in the POWER_OFF_l initial state, in which all components are off. This eliminates discharging the energy storage $\underline{9}$ by the accumulator $\underline{11}$ or by active appliances of the onboard network $\underline{23}$ when the ignition is off. The system then switches 
from the POWER_OFF_1 state to POWER_ON_2 provided the condition of the ignition being on is fulfilled. The system remains in this state unless one of the two possible conditions is met - with the following priority:

- Ignition switched off, the system returns to the initial POWER_OFF_l state.

- Starter activated, the system goes to the ENGINE_START_3 state.

In the POWER_ON_2 state, the system charges the energy storage $\underline{9}$ with electric current acceptable for the current state of accumulator 11 . It then goes from the POWER_ON_2 state to ENGINE START 3 provided that storage $\underline{9}$ is charged and the starter activated. If the accumulator 11 voltage drops to below the acceptable limit, or the ignition is switched off during the process of storage $\underline{9}$ charging, the system goes to the POWER_OFF_1.

In the ENGINE_START_3 state, the system arranges controlled starter powering from the electricity storage $\underline{9}$ or from the accumulator $\underline{11}$. The ENGINE_START_3 state is terminated if one of three conditions is met - in the following priority order:

- Ignition switched off, the system returns to the initial POWER_OFF_l state.

- Starter switched off and engine not running, the system returns to the POWER_ON_2 state.

- Starter switched off and engine running, the system goes to the COLD_ENGINE_4 state.

Controlled connecting and disconnecting of the electricity storage 9 takes place while in the COLD_ENGINE_4 state. This state is terminated under the following conditions:

- Ignition switched off, the system returns to the initial POWER_OFF_l state.

The required operating temperatures of the engine 1 and other necessary components affecting engine $\underline{1}$ operation:

- $\quad$ are reached. If the engine runs in the range of idle rpm, the control goes tothe IDLING_5 state.

- The required operating temperatures of the engine 1 and other necessary components affecting engine 1 operation are reached. If the engine runs outside the range of idle rpm, the control goes to the STEADY_DRIVE_7 state.

In the IDLING_5 state, the system controls the electronic air-conditioning $\underline{13}$ and the main electric motor $\underline{6} \mathrm{c}$. The electronic air-conditioning 13 management takes place with respect to minimizing the combustion engine 1 load, however, provided that the condition of maintaining the set temperature in the vehicle is met. The main electric motor is controlled in such a way as to minimize mechanical losses.

The idling state is terminated by:

- Ignition switching off, the system returns to the initial POWER_OFF_1 state.

- Vehicle acceleration, when the system goes to the ACCELERATION_6 state.

In the ACCELERATION_6 state, the system operates the electronic air-conditioning 13 , the auxiliary generator $7 \mathrm{a}$, the auxiliary electric motor $7 \mathrm{c}$, the main generator $6 \mathrm{a}$ and the main electric motor $\underline{6 \mathrm{c}}$ in such a way as to utilize the energy previously accumulated in the storage 
$\underline{9}$ and the accumulator 11 as much as possible, as well as to accelerate the vehicle by means of the main electric motor $\underline{6 c}$ and to increase the combustion engine dynamics by means of the auxiliary electric motor $\underline{7 \mathrm{c}}$.

The acceleration state is terminated by:

- The vehicle settling into steady driving, the system returns to the STEADY_DRIVE_7 state.

- Vehicle deceleration, when the system goes to the DECELERATION_8 state.

In the STEADY_DRIVE_7 state, the system controls the electronic air-conditioning $\underline{13}$, the auxiliary generator $7 \mathrm{a}$ and the main generator $\underline{6 \mathrm{a}}$ in such a way as to fulfill the operator's requirements on the pre-set temperature and to guarantee the minimum off-take of electricity generated in the auxiliary generator $\underline{7 \mathrm{a}}$ and the main generator $\underline{6 \mathrm{a}}$ and accumulated in the electricity storage $\underline{9}$ and the accumulator $\underline{11}$.

The steady driving state is terminated by:

- Vehicle acceleration, when the system goes to the ACCELERATION_6 state.

- Vehicle deceleration, when the system goes to the DECELERATION_8 state.

In the DECELERATION_8 state, the system controls the electronic air-conditioning $\underline{13}$, the auxiliary generator $\underline{7 \mathrm{a}}$ and the main generator $\underline{6 \mathrm{a}}$ in such a way as to provide the maximum possible utilization of the braking energy for the electronic air-conditioning $\underline{13}$, for charging firstly the electricity storage $\underline{9}$ and the onboard accumulator $\underline{11}$.

The deceleration state is terminated by:

- The vehicle settling into steady driving, the system returns to the STEADY_DRIVE_7 state.

- Vehicle acceleration, when the system goes to the ACCELERATION_6 state.

\section{CONCLUSION}

The invention is further clarified in diagrams, where Fig. 1 represents a block diagram of V.E.M.S. and its connection to the combustion engine in the basic arrangement, and Fig. 2 represents the state diagram of its behavior. This is the result of long-term collaboration between academic laboratories (university departments) and an innovative company specializing in the manufacture of internal combustion engines.

\section{REFERENCES}

Internal documents of the MSR Engines Ltd. 